Revista Internacional de

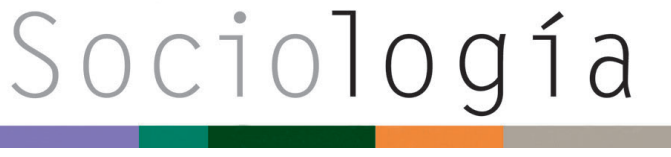

\section{POSICIÓN EN EL HOGAR Y GÉNERO. Desigualdades en la calidad de vida relacionada con la salud entre la población adulta en España}

JORDI GUMÀ

jguma@ced.uab.es

Rocio TREVIÑo

rtrevino@ced.uab.es

Antonio D. CÁmara

Universidad Autónoma Barcelona, España

adcamara@ced.uab.es

Como citar este artículo / Citation: Gumà, J. y R. Treviño. 2015. "Posición en el hogar y género. Desigualdades en la calidad de vida relacionada con la salud entre la población adulta en España", Revista Internacional de Sociología, 73, http://dx.doi.org/103989/2013.03.04

\section{RESUMEN}

Este artículo explora la asociación entre la posición en el hogar y la calidad de vida relacionada con la salud (CVRS) entre la población de 30-64 años residente en España ( $N=13.258)$ con una perspectiva de género. Se utilizan modelos multivariables de regresión lineal independientes según sexo a partir de la muestra española de la Encuesta Europea de Salud de 2009 incluyendo las siguientes variables de control: edad, relación con la actividad, nivel educativo, capital social, nacionalidad y variables de salud objetiva. Los resultados muestran que la posición en el hogar es más determinante sobre la CVRS que los indicadores de estatus individual entre la población femenina, lo que no ocurre entre la población masculina. La monoparentalidad es la posición del hogar asociada a una peor CVRS, penalizando en mayor medida a las mujeres. Entre los hombres, la CVRS se muestra más sensible a la convivencia o no en pareja. Se discutirán, pues, los determinantes relacionados con el género.

\section{Palabras Clave}

Calidad de vida; Determinantes sociales de la salud; Género; Hogar.
Revista Internacional de Sociología RIS

DOI: http://dx.doi.org/10.3989/ris.2013.03.04 vol. 73 (1), e003, enero-abril, 2015, ISSN-L: 0034-9712
POSITION AT HOME AND GENDER. Quality of life inequalities regarding the Spanish adult population health
Copyright: @2015 CSIC. Este artículo de acceso abierto distribuido bajo los terminos de la licencia Creative Commons Attribution-Non Commercial (by-cn) Spain 3.0.

Recibido: 04/03/2013. Aceptado: 20/09/2014

\section{AbSTRACt}

This paper applies a gender perspective to explore the relationship between the position of individuals within the household and the health-related quality of life (CVRS) among the population aged 30 to 64 living in Spain $(N=13.258)$. Microdata from the Spanish sample of the European Health Survey of 2009 are utilized for this purpose. Multivariate linear regression models for males and females are run which include the following controllers: age, labor status, educational level, social capital, nationality and variables approaching individuals' objective health. Results show that, among females, the position within the household is more influential on CVRS than some other individual characteristics. This is not the case among males. One-parent family is the household position that penalize CVRS the most which stands especially for females. Among males, living (or not) in couple is the most influential factor on CVRS. Gender-related determinants are discussed.

\section{KEYWORDS}

Gender; Health-Related Quality of Life; Household; Social Determinants of Health. 


\section{INTRODUCCIÓN}

El descenso de la mortalidad en los países desarrollados se ha producido sin una reducción paralela de las desigualdades de salud (Marmot, 2005; Stephens, 1998). Por tales desigualdades se entienden aquellas diferencias de salud injustas, evitables y sistemáticas (Organización Mundial de la Salud 2007) cuyos determinantes sociales han sido objeto preferente de estudio por parte de las ciencias sociales.

Entre los diversos determinantes de la salud analizados, nuestro interés se centra en explicar las desigualdades de salud mediante el hogar (Joutsenniemi 2007; Hughes y Waite 2002), entendido como un contexto común de convivencia pero también como un espacio de diferenciación en relación con los recursos, responsabilidades y oportunidades sociales de sus miembros. En este sentido, la posición en el hogar define el lugar del individuo, no solo en relación con su estructura (hogar unipersonal, hogar pluripersonal), sino también en relación con su situación en el núcleo o núcleos familiares del hogar (como miembro de la pareja, como padre o madre emparejados, como hijo/a, como madre o padre monoparental, fuera de un núcleo familiar). Las relaciones dentro del hogar se articulan en un intercambio continuo y diario de recursos económicos, de soporte emocional, de cuidado, de información, etc. (Hughes y Waite 2002). Estos intercambios de recursos operan en función de unas normas de interacción entre hombres y mujeres basadas en consensos socioculturales que definen la formación histórica y social de los contratos de género (Hirdman 1998), articulados siempre en torno a la división sexual del trabajo en la esfera productiva y la doméstica. Balances desequilibrados en los intercambios y relaciones descritos son susceptibles de traducirse en desigualdades en salud.

La aproximación a estas desigualdades en salud se realiza a partir del indicador complejo de calidad de vida relacionada con la salud (CVRS), el cual permite captar con mayor detalle las diferencias de salud (Bowling 1999) de la población adulta (en este trabajo representada por las edades entre 30-64), donde los perfiles son más homogéneos que entre la población mayor (habitualmente representada por las edades de 65 años y más). Aunque el concepto general de calidad de vida relacionada con la salud no tiene una definición única debido a su naturaleza subjetiva, sí puede destacarse que prima la valoración de cómo la salud condiciona el día a día de los individuos (Bullinger et al. 1993), ubicándose dentro de la dimensión subjetiva de la salud. Además, refleja la diferencia entre salud experimentada y esperada (Carr et al. 2001). Se trata, por tanto, de un concepto multidimensional que aglutina información sobre la salud mental, física y social, teniendo en cuenta el efecto que sobre la salud tienen, tanto las características sociodemográficas propias del individuo, como algunos de los elementos externos que interaccionan con estos (Vilagut et al. 2008; Anguita et al. 2001; Bowling 1999). De este modo se ajusta a la definición multidimensional de la salud dada por la Organización Mundial de la Salud'.

El objetivo de este trabajo es explorar con una perspectiva de género la asociación entre la posición en el hogar y las desigualdades de salud, medidas a través de la CVRS, de los individuos de 30 a 64 años de edad. Este análisis se realiza controlando por determinantes sociales cuya relación con las desigualdades de salud ha sido probada anteriormente (estatus socioeconómico, capital social), factores sociodemográficos (edad y nacionalidad) e indicadores de salud objetiva (número de enfermedades crónicas y dolor físico) de los individuos. Para ello se han analizado los microdatos de la muestra española de la Encuesta Europea de Salud de 2009. Los indicadores de salud objetiva han sido incluidos para poder estandarizar el efecto de la posición en el hogar sobre un indicador subjetivo como la CVRS, relacionando ambas dimensiones de la salud y permitiendo de este modo aproximar el efecto a igual situación de salud objetiva de los determinantes sociales y de los factores demográficos introducidos en el análisis multivariable.

El rango de edad estudiado (30-64) tiene como objetivo acotar una población con mayor grado de homogeneidad en cuanto al ciclo laboral y familiar, y en la que los efectos de las desigualdades de género sobre la salud pueden resultar más evidentes. Debe destacarse, asimismo, el valor añadido que representa el estudio de poblaciones en edades en las cuales las políticas de salud pública pueden actuar para revertir situaciones desaventajadas de salud.

En primer lugar se presentan los antecedentes del tema de estudio, y a continuación un segundo apartado donde se recogen la fuente de datos utilizada, la descripción de las variables y el método de análisis. Los resultados se dividen en dos partes: en la primera se describe el perfil de la posición en el hogar por sexo y edad de la población objeto de estudio y los valores medios del indicador de CVRS; en la segunda se aborda el análisis multivariable de regresión lineal realizado para obtener el valor neto de la asociación entre la posición en el hogar y la CVRS.

\section{AnteCEDentes}

Los determinantes sociales de la salud permiten entender por qué situaciones iniciales de salud objetiva similares acaban evolucionando en patrones de salud desiguales entre individuos a lo largo de su vida (Marmot 2005). Estos determinantes son conocidos también como "las causas de las causas" (Rose 1992) debido a su efecto indirecto

\footnotetext{
${ }^{1}$ Salud es un estado de bienestar completo tanto físico, mental y social y no simplemente la ausencia de enfermedad o discapacidad (Organización Mundial de la Salud 1946).
} 
sobre la salud (McKinlay 1993) derivado de su vínculo con los diferentes niveles de exposición a los riesgos de salud a lo largo del ciclo vital.

Entre estos determinantes, la situación de pareja de los individuos ha constituido una primera aproximación al estudio de la asociación de las relaciones familiares en el hogar con la salud. Existe un amplio consenso en señalar que la convivencia en pareja tiene un efecto positivo e inmediato sobre la salud de los hombres y, aunque de menor intensidad, también sobre las mujeres a medio y a largo plazo (Lillard y Panis 1996; Waite 1995). Estos beneficios de la convivencia en pareja han sido explicados a partir de tres factores: 1) por la reducción de los comportamientos de riesgo y de los hábitos no saludables a que va asociada (Lillard y Waite 1995); 2) porque deriva en la creación y mantenimiento de una red social de soporte a la que recurrir en situaciones críticas (Waite 1995); y, finalmente, 3) por el incremento del bienestar material que implica la vida en pareja como resultado de una economía de escala que permite la combinación de recursos y la especialización de tareas entre los miembros de la pareja (Lillard y Panis 1996).

Posteriormente se ha ampliado el rango de relaciones del hogar contempladas en los análisis de las desigualdades en salud incluyendo también la estructura del hogar y abordando en la mayoría de los estudios las dimensiones subjetivas y mentales de la salud (Joutsenniemi 2007; Hughes y Waite 2002). Estos trabajos han mostrado que los hombres que residen en estructuras familiares que implican una convivencia en pareja muestran una mejor salud, mientras que en las mujeres el nivel de salud depende de la carga familiar a la cual se ven expuestas (Joutsenniemi 2007; Martikainen et al. 2005). También ha sido señalado que las situaciones ventajosas de salud pueden revertirse por algún cambio familiar de tipo traumático (separación o defunción de la pareja), con un efecto negativo mayor en función de la proximidad temporal a la experimentación de este (Hughes y Waite 2002).

Los estudios mencionados han optado por abordar la relación entre hogar y salud a través de una perspectiva contextual del hogar, de manera común para todos sus miembros, sin considerar que el reparto de responsabilidades y la "carga familiar" se ven diferenciados en función del género, la edad y las relaciones familiares de los miembros que componen el hogar (por ejemplo, los hijos/ as jóvenes son mayoritariamente receptores de recursos mientras que sus padres-madres son proveedores). Es más, la dinámica de género interactúa junto con la edad y el ciclo de vida estructurando posiciones diferenciadas en el hogar que condicionan el día a día de los individuos, y por tanto, son susceptibles de influir en las desigualdades de salud entre estos. En este sentido, la incompleta revolución de género (McDonald 2000; Goldsheider 2000) experimentada en los países occidentales, que ha hecho que sea mucho más probable que las mujeres participen en el mercado de trabajo laboral a que los hombres asuman sus responsabilidades de trabajo doméstico y de cuidado (ibíd.), ha contribuido a que las primeras acumulen mayor carga de trabajo total (trabajo laboral, trabajo doméstico y de cuidado) (Durán 1998) y al fenómeno de la doble presencia femenina (Balbo 1978) que ha sido asociado a situaciones de frustración y estrés (Rosales 2003). Otros procesos sociodemográficos que han afectado a la carga total de trabajo de los hogares y a su redistribución de género han sido el envejecimiento de la población, el retardo de la emancipación ligado a la expansión educativa o el incremento del divorcio. Así, por ejemplo, Spijker y Pérez (2010) señalan que la presencia de una persona dependiente en el hogar "tradicionaliza" los roles de género en el hogar.

Desde este punto de vista, la posición en el hogar se configura como una aproximación a la estructuración de los roles de género, no solo en función del sexo, sino también de la edad y del ciclo de vida de los individuos, permitiendo ajustar los patrones de intercambio en la oferta y demanda de recursos del hogar (económicos, de cuidado y soporte emocional, de información, etc. (Hughes y Waite 2002) que condicionan la vida cotidiana de los individuos. Cuando se produce una situación de desequilibrio en este tipo de intercambios se suele observar una desigualdad de género en, por ejemplo, la práctica de actividad física, la disposición de tiempo libre, el número de horas de sueño, etc. (Observatorio de Salud de la Mujer 2005).

Pero se debe tener en cuenta que la dinámica de género interactúa en todos los determinantes sociales de la salud generando 1) un desigual grado de exposición a los diferentes riesgos que afectan a la salud 2) una percepción de la propia salud también diferenciada; 3) y finalmente, diferencias en la asistencia sanitaria recibida. En relación con el primer punto, cabe destacar que el conjunto de determinantes sociales configura unos estilos de vida diferenciados que se vinculan a las definiciones de masculinidad y feminidad (Maclnnes 1998; García-Mina 2003), que ejercen un efecto directo tanto sobre la salud como incluso sobre la supervivencia de los individuos. En relación con el segundo punto, los hallazgos de diversos trabajos han mostrado diferencias en la autopercepción de la salud de hombres y mujeres ante síntomas de similar naturaleza, llegando a plantear la posibilidad de que la mayor prevalencia de la morbilidad crónica en las mujeres tenga como origen la capacidad de reconocer o verbalizar las enfermedades por parte de estas y no tanto como consecuencia de diferencias biológicas (Observatorio de Salud de la Mujer 2005). Por lo que respecta al tercer punto, el sesgo de género en la práctica diagnóstica (Ruiz y Verdú 2004) consistente en la extrapolación a las mujeres de resultados diagnósticos obtenidos a partir de ensayos clínicos realizados en su mayoría con hombres como sujetos de estudio, ha llevado a tomar la idea de que la sintomatología de 
las diversas enfermedades es la misma en ambos sexos, provocando imprecisiones en la definición de los procesos de prevención, tratamiento y atención de dichas enfermedades en el caso femenino (Griffith 2005; Observatorio de Salud de la Mujer 2005).

Además, para obtener una visión global sobre los patrones de salud diferenciados según sexo se deben añadir a las desigualdades de género aquellas diferencias cuyo origen es de naturaleza biológica. Cuando se examina el perfil de salud objetiva de ambos sexos, entre los hombres las enfermedades cardiovasculares son más prevalentes que entre las mujeres, mientras que en las mujeres se observan altas prevalencias de enfermedades mentales y patologías del sistema músculo-esquelético (Oksuzyan 2009; Arber y Cooper 1999). Este último punto constituye una de las explicaciones más utilizadas para justificar las diferencias de salud por sexo: las enfermedades masculinas tienen una mayor relación con la supervivencia mientras que las femeninas mostrarían un mayor recorrido temporal y, por lo tanto, derivarían en un progresivo desgaste de la salud. Sin embargo, resulta imposible disociar completamente el efecto de las desigualdades de género descritas anteriormente sobre las diferencias de salud entre sexos.

Para concluir este apartado destacaremos algunas de las evidencias empíricas que justifican la inclusión del resto de covariables del trabajo, referidas a diferentes determinantes sociales y demográficos de la salud.

El determinante social de la salud que ha recibido mayor atención es el estatus socioeconómico individual aproximado a partir de la relación con la actividad de los individuos, la situación profesional, el nivel educativo o el nivel de pobreza, entre otros. Todos los trabajos revisados han mostrado inequívocamente que este factor tiene un gran peso a la hora de definir el sentido y la intensidad de las desigualdades de salud, mostrando mejor salud aquellas personas con un estatus socioeconómico superior (Marmot 2005; Borrell et al. 2004). Los mecanismos que operan en ese sentido aluden no solo al impacto positivo sobre la salud de la mayor disposición de recursos, sino también al mayor conocimiento sobre cómo llevar una vida más saludable (dieta, consumo de tabaco, alcohol, estupefacientes, etc.) asociado a mayores niveles educativos (Marmot 2005).

El capital social, entendido como el conjunto de relaciones bidireccionales que se establecen entre los individuos y sus círculos más próximos, tanto familiares como extrafamiliares, es otro de los determinantes que mayor interés ha suscitado. Su asociación con el estado de salud de los individuos, sobre todo en su dimensión subjetiva, ha sido contrastada ampliamente (Puga et al. 2007; Berkman et al. 2000; Rogers 1996). Las relaciones sociales se han mostrado beneficiosas para la salud de los individuos por: 1) la ayuda directa que canalizan en caso de necesidad de cuidado; 2) por el efecto disuasorio que ejerce el control social en la práctica de actividades de riesgo (consumo de sustancias, conducción en estado de embriaguez, etc.); 3) porque implican un mayor nivel de integración social que deriva en una vía de acceso a recursos (oportunidades de trabajo, cuidado sanitario, etc.) así como en un sentimiento de pertenencia a un colectivo. Estos beneficios se traducen en una menor erosión de la salud general así como en efectos positivos sobre la salud mental (aumento de la autoestima, descenso de los síntomas de depresión e incremento de la sensación de bienestar) (Berkman et al. 2000; Rogers 1996).

La nacionalidad de los individuos se ha contemplado en nuestro trabajo debido a la diversificación que la población residente en España experimentó durante la primera década del siglo XXI como consecuencia de los intensos flujos inmigratorios. Aparte de las diferencias potenciales en las condiciones y el estado de salud como resultado del efecto acumulado de los ambientes de origen hasta el momento del movimiento migratorio, la nacionalidad es una fuente de diferenciación de derechos. La inmigración se asocia a situaciones de desventaja en los ámbitos laboral, social y personal (Porthé et al. 2006), y por tanto, a escenarios de mayor vulnerabilidad social que pueden acabar afectando a la salud. Diferentes estudios han destacado el efecto positivo del estatus de inmigrante sobre la salud al ser seleccionados en el origen los individuos en condiciones lo suficientemente ventajosas como para emprender la migración (Abraído-Lanza et al. 1999; Swallen 1997). Sin embargo, otros trabajos han encontrado en nuestro país que, incluso en el caso de existir esta selección previa, las peores condiciones de vida a las cuales se ven abocados los inmigrantes en la mayoría de los casos llevan a que esta posible ventaja inicial desaparezca después de pocos años de permanencia en el país de destino (GarcíaGómez y Oliva 2009; Solé y Rodríguez 2010).

Finalmente, la edad es clave por el deterioro de la salud durante el proceso de envejecimiento, su efecto sobre la manera de autopercibir la propia salud y su vínculo con la posición que se ocupa en el hogar a través del ciclo familiar. Así, además de su más que probada asociación con los perfiles de salud (peor salud en edades más avanzadas), resulta también fundamental a la hora de establecer las referencias que toma un individuo a la hora de autodeclarar su salud, ya que todos acostumbramos a compararnos con otros individuos de nuestro mismo grupo de edad (Gumà 2009; Idler 1993). Por tanto, un mismo problema de salud será valorado de manera más o menos negativa dependiendo de si es más o menos frecuente en el grupo de edad de referencia. La edad también se asocia al ciclo vital de las personas, permitiendo de este modo distinguir entre situaciones más o menos frecuentes (no es lo mismo ocupar la posición de hijo/a con una edad de 15 años que a los 40 años). 


\section{Fuentes Y MÉTOdOS}

Los datos utilizados proceden de la muestra española de la Encuesta Europea de Salud (EES) de 2009. La EES es un proceso estadístico armonizado en el marco del Sistema Estadístico Europeo cuyo objetivo es recoger información sobre diferentes dimensiones de la salud y sus determinantes sociales. La unidad de muestreo fueron los hogares dentro del ámbito geográfico español, entrevistándose a un total de 22.188 individuos residentes en esos hogares.

Para este trabajo se han seleccionado las edades 30 64 que suman 13.258 individuos: $6655(50,2 \%)$ hombres y $6603(49,8 \%)$ mujeres.

\section{Calidad de vida relacionada con la salud}

El indicador de calidad de vida relacionada con la salud (CVRS), nuestra variable dependiente, consiste en una escala aditiva resultante de sumar los valores de las respuestas dadas a una batería de nueve preguntas incluidas en el cuestionario de la EES. Estos nueve ítems (Tabla 1) son los únicos incluidos en el cuestionario general de la EES del conjunto de preguntas pertenecientes al llamado instrumento SF-36 (Instituto Nacional de Estadística 2009). Los ítems recogen información sobre la percepción de cuatro dimensiones de la calidad de vida relacionada con la salud: el impacto emocional, el cansancio psicológico, la vitalidad y el estado de ánimo de los individuos. Las posibilidades de respuesta a estos ítems son: "siempre", "casi siempre", "algunas veces", "solo alguna vez" y "nunca", tomando como referencia las cuatro semanas anteriores a la entrevista. A cada una de estas categorías se le ha asignado una puntuación entre 1 y 5 , siendo 1 la connotación más negativa y 5 la más positiva en cada caso (Tabla 1). Las últimas dos columnas de la tabla 1 se refieren al porcentaje de respuesta entre las edades 30 y 64 .

El indicador final recoge la suma de las nueve puntuaciones para cada individuo, oscilando por tanto los valores dentro de un rango de 9 a 45 puntos. No poder contar con el resto de preguntas del instrumento SF-36 ha impedido utilizar los pesos estándar para las diferentes dimensiones recogidas por los nueve ítems, razón por la cual todos los ítems tienen el mismo peso en el indicador final. Para facilitar su interpretación, el valor obtenido ha sido convertido a una escala entre 0 y 100 mediante la siguiente fórmula:

Fórmula 1

$$
\frac{\sum \text { respuestas }-9}{36} * 100
$$

\section{Determinantes sociales de la salud}

Posición en el hogar

Esta variable combina información sobre el hogar y el núcleo familiar. El hogar lo conforman las personas que

Tabla 1.

Descripción ítems utilizados para elaborar el indicador de CVRS.

\begin{tabular}{|c|c|c|c|c|c|c|c|}
\hline & \multicolumn{5}{|c|}{ Respuestas posibles } & \multicolumn{2}{|c|}{$\%$ Respuesta } \\
\hline & Siempre & $\begin{array}{c}\text { Casi } \\
\text { siempre }\end{array}$ & $\begin{array}{c}\text { Algunas } \\
\text { veces }\end{array}$ & $\begin{array}{c}\text { Sólo } \\
\text { alguna vez }\end{array}$ & Nunca & Hombres & Mujeres \\
\hline Se sintió lleno de vitalidad & 5 & 4 & 3 & 2 & 1 & $99,5 \%$ & $99,7 \%$ \\
\hline Estuvo especialmente tenso & 1 & 2 & 3 & 4 & 5 & $99,5 \%$ & $99,7 \%$ \\
\hline Se sintió tan bajo de moral que nada podía animarlo & 1 & 2 & 3 & 4 & 5 & $99,5 \%$ & $99,7 \%$ \\
\hline Se sintió calmado y tranquilo & 5 & 4 & 3 & 2 & 1 & $99,6 \%$ & $99,7 \%$ \\
\hline Tuvo mucha energía & 5 & 4 & 3 & 2 & 1 & $99,6 \%$ & $99,6 \%$ \\
\hline Se sintió desanimado y deprimido & 1 & 2 & 3 & 4 & 5 & $99,2 \%$ & $99,5 \%$ \\
\hline Se sintió agotado & 1 & 2 & 3 & 4 & 5 & $99,4 \%$ & $99,6 \%$ \\
\hline Se sintió feliz & 5 & 4 & 3 & 2 & 1 & $99,3 \%$ & $99,3 \%$ \\
\hline Se sintió cansado & 1 & 2 & 3 & 4 & 5 & $99,4 \%$ & $99,6 \%$ \\
\hline
\end{tabular}

Fuente: Cuestionario de la EES 2009 para España (Instituto Nacional de Estadística). 
residen habitualmente en la misma vivienda. El núcleo familiar corresponde por definición a: 1) una pareja sin hijos; 2) una pareja con algún hijo; 3) una madre sin pareja y con algún hijo (monoparental femenina); 4) un padre sin pareja con algún hijo (monoparental masculino). A su vez, la posición en el núcleo distingue cuatro situaciones: en pareja sin hijos, en pareja con algún hijo, padre o madre solo/a, posición de hijo/a en un núcleo. La construcción de núcleos ha priorizado la relación paterno/materno-filial más joven en aquellos hogares donde se observa la convivencia entre tres o más generaciones. De este modo, la posición en el hogar diferencia las cuatro posiciones de núcleo arriba mencionadas. Además, distingue aquellos individuos que están en pareja en función de sí tienen uno o más hijos y, por último, se añaden la de hogar unipersonal y otras situaciones. En esta última categoría se ubican aquellos individuos que están fuera de núcleo en un hogar, pudiendo tener o no relación de parentesco con algún miembro de los núcleos del hogar si los hay. La posición de padre o madre sin pareja, es decir, encabezando un núcleo monoparental, no se diferencia según el número de hijos debido al bajo número, tanto absoluto (61 hombres y 443 mujeres) como relativo (un 0,9\% entre los hombres y un $6,7 \%$ entre las mujeres) de individuos que se encontraban en esta situación en la muestra de la EES.

\section{Estatus socioeconómico}

El estatus socioeconómico ha sido aproximado mediante dos variables introducidas de manera independiente: la relación con la actividad y el nivel de estudios. La relación con la actividad combina la situación en relación con el empleo y la situación profesional. Los individuos no ocupados se dividen en: "desempleado/a", "dedicación principal a las labores domésticas", e "inactivo/a". Los ocupados se dividen en: "asalariados/as por cuenta ajena", "empresarios/as con o sin empleados", y "otras situaciones de ocupación". El nivel educativo hace referencia al máximo alcanzado en el momento de la entrevista, proponiéndose las siguientes tres categorías: "educación obligatoria o inferior" (individuos que como máximo han acabado estudios primarios obligatorios), "educación secundaria no obligatoria" y "estudios universitarios".

\section{Capital Social}

La covariable "capital social" se deriva de la respuesta a la pregunta del cuestionario de la EES: "En caso de tener un problema personal grave de cualquier tipo ¿con cuántas personas cercanas a usted podría contar?". Las posibles respuestas fueron agrupadas en dos categorías: "dos o menos" (bajo capital social) y "tres o más" (alto capital social). Esta agrupación se debe a que únicamente el $1,7 \%$ de los entrevistados entre 30 y 64 años declararon no tener ninguna persona a la cual recurrir en caso de un problema personal grave, optándose por expandir el criterio de definición de bajo capital social hasta la siguiente categoría de respuesta (una o dos).

\section{Variables de control}

La variable "edad" se introduce de forma continua para los individuos con edades comprendidas entre los 30 y 64 años.

La "nacionalidad" diferencia si los individuos tienen nacionalidad española y, entre los de nacionalidad extranjera, los de la UE-27 y los que de fuera de la UE-27. De este modo, se establecen tres grandes grupos diferenciados en función de sus derechos civiles.

Aunque el indicador de CVRS se ubica dentro de la dimensión subjetiva de la salud, esta última no es independiente de la dimensión objetiva, mostrando los indicadores subjetivos una consistente relación y capacidad predictiva sobre la evolución inmediata de los indicadores objetivos (Schneider et al. 2004). Por este motivo se han introducido dos variables de salud objetiva para estandarizar el efecto de la posición en el hogar sobre un indicador subjetivo como la CVRS a igual situación de salud objetiva.

La primera variable de salud objetiva recoge el número de enfermedades crónicas diagnosticadas por un profesional de la medicina. Dado que la tipología de enfermedades diagnosticadas es muy amplia, se ha optado por tener en cuenta la acumulación de estas enfermedades crónicas. Con el objetivo de evitar trabajar con categorías con un bajo número de casos, se ha utilizado la siguiente categorización: "ninguna", "una", y "dos o más" enfermedades crónicas.

También se ha querido controlar el efecto que el padecimiento de un dolor físico en un momento temporal cercano a la entrevista puede tener sobre las respuestas a los ítems del indicador de CVRS. Para eso se incluye una variable derivada que combina los ítems: "¿Durante las 4 últimas semanas ha padecido algún tipo de dolor 0 molestia física?" y "¿Qué grado de dolor o molestia física ha tenido?". Tal y como se deduce del enunciado literal de las preguntas, se recogen aquellas situaciones que producen dolor y que no tienen que ser necesariamente de larga duración como en el caso de las enfermedades crónicas. De este modo, la variable final contempla las siguientes posibilidades: "no ha experimentado dolor/molestia"; "ha experimentado dolor/molestia leve o moderado"; y "ha experimentado dolor/molestia severo o extremo".

Al incluir como variables de control dos ítems vinculados a la salud objetiva, el número de enfermedades crónicas diagnosticadas y el padecimiento e intensidad de un dolor en el periodo previo a la entrevista, se realizó un análisis previo de correlación entre las mismas. El coeficiente Tau de Kendall mostró que la correlación era de un nivel suficientemente bajo para que ambas fueran introducidas como variables independientes en el mismo modelo (Tau de Kendall= 0,324). 


\section{MÉTOdO}

Trabajos previos con el indicador de CVRS mostraron que seguía una escala de intervalo (Vilagut et al. 2008; Anguita et al. 2001) y por tanto se ha optado por utilizar el método estadístico de regresión lineal para el análisis multivariable en nuestro trabajo, introduciendo como variables dummy (valores 001 ) todas las categorías de las variables independientes analizadas, a excepción de la edad que ha sido introducida como una variable continua. La distribución del indicador de CVRS, muestra que el $90 \%$ de los casos se concentra en la segunda mitad del rango (50-100) (Figura 1). El hecho de contar con una escala de valores de este rango confiere una mayor sensibilidad al indicador para aproximar las desigualdades de salud de la población adulta. Esta población en el caso de variables como por ejemplo la salud autopercibida, con únicamente cuatro o cinco posibilidades de respuesta, muestra un alto porcentaje de concentración de sus respuestas en las valoraciones positivas de la salud, reduciendo mucho la variabilidad entre individuos.

Las desigualdades de CVRS fueron primeramente analizadas mediante un único modelo para hombres y mujeres, obteniendo diferencias significativas entre ambos (coeficiente de $-3,3$ de las mujeres respecto a los hombres; Tabla 1 del Anexo). No obstante se decidió basar el análisis e interpretación final de los resultados en modelos diferenciados para hombres y mujeres que permitiesen ver el sentido de la asociación de las covariables de manera independiente, permitiendo captar de este modo los potenciales efectos de las desigualdades de género.

\section{Resultados}

\section{Posición dentro del hogar}

La tabla 2 presenta el perfil por edad y sexo de la posición en el hogar que puede considerarse una aproximación transversal al ciclo familiar de los individuos.

Entre los hombres, las posiciones en el hogar que implican la no convivencia en pareja o con hijos, como son vivir solo u ocupar la posición de hijo dentro del núcleo, presentan valores agregados menores con la edad. Esto se debe al descenso del número de individuos que ocupan la posición de hijo, mientras que en el caso de los hogares unipersonales los porcentajes muestran un ligero incremento en edades más avanzadas. En cuanto a las tres posiciones en el hogar en las cuales se convive en pareja (núcleo sin hijos, núcleo con un hijo/a y núcleo con dos o más hijos), su valor porcentual es mayor en los grupos de edad cercanos a 65 (69\% en el grupo 30-34 mientras que en el grupo $60-64$ es un $83 \%$ ). Este mayor peso relativo de los hombres que conviven en pareja por edad se da de manera paralela al incremento de la convivencia con hijos por edad, que llega a su máximo valor porcentual en el grupo de edad 40-49 (67,5\%). En cambio, se observan valores menores entre las edades más avanzadas (por ejemplo, el porcentaje más bajo se observa en el grupo de edades 60-64, con un 34\%). Por último, la monoparentalidad entre los hombres muestra ser una posición marginal, incluso con menor peso relativo que las otras posiciones en el hogar.

Figura 1.

Distribución del indicador de CVRS. España 2009.

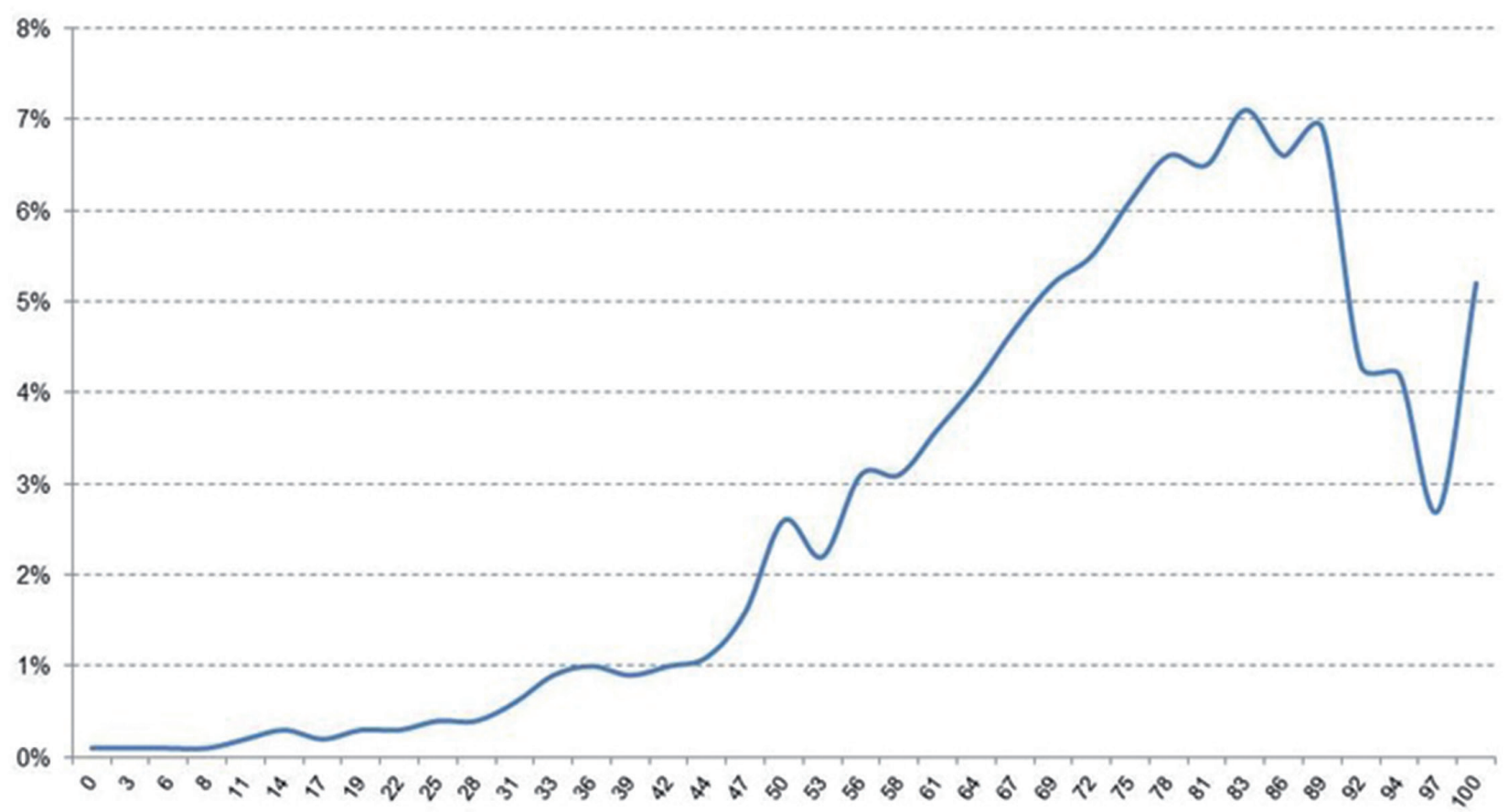

Fuente: Cuestionario de la EES 2009 para España (Instituto Nacional de Estadística). 
Tabla 2.

Tipologías de posición en el hogar según sexo y grupos de edad. España 2009.

\begin{tabular}{lcccccccc}
\hline & \multicolumn{4}{c}{ Hombres } & \multicolumn{4}{c}{ Mujeres } \\
\cline { 2 - 9 } & $\mathbf{3 0 - 3 9}$ & $\mathbf{4 0 - 4 9}$ & $\mathbf{5 0 - 5 9}$ & $\mathbf{6 0 - 6 4}$ & $\mathbf{3 0 - 3 9}$ & $\mathbf{4 0 - 4 9}$ & $\mathbf{5 0 - 5 9}$ & $\mathbf{6 0 - 6 4}$ \\
\hline Vivir solo/a & $8,1 \%$ & $6,7 \%$ & $7,7 \%$ & $10,1 \%$ & $4,3 \%$ & $4,4 \%$ & $8,7 \%$ & $13,3 \%$ \\
Posición hijo/a en el núcleo & $16,7 \%$ & $10,2 \%$ & $3,6 \%$ & $1,9 \%$ & $13,6 \%$ & $4,7 \%$ & $4,1 \%$ & $2,9 \%$ \\
Miembro pareja sin hijos & $20,3 \%$ & $10,9 \%$ & $26,5 \%$ & $49,4 \%$ & $16,5 \%$ & $10,4 \%$ & $29,2 \%$ & $49,1 \%$ \\
Miembro pareja con un hijola & $24,1 \%$ & $21,8 \%$ & $27,1 \%$ & $24,2 \%$ & $25,8 \%$ & $22,8 \%$ & $25,7 \%$ & $17,8 \%$ \\
Miembro pareja con dos o más hijos & $24,6 \%$ & $45,7 \%$ & $31,0 \%$ & $9,8 \%$ & $31,1 \%$ & $45,4 \%$ & $19,2 \%$ & $7,5 \%$ \\
Miembro núcleo monoparental & $0,3 \%$ & $1,1 \%$ & $1,4 \%$ & $1,3 \%$ & $4,3 \%$ & $8,3 \%$ & $9,1 \%$ & $4,8 \%$ \\
Otra posición hogar & $5,8 \%$ & $3,6 \%$ & $2,8 \%$ & $3,3 \%$ & $4,4 \%$ & $3,9 \%$ & $4,0 \%$ & $4,5 \%$ \\
Total & $100 \%$ & $100 \%$ & $100 \%$ & $100 \%$ & $100 \%$ & $100 \%$ & $100 \%$ & $100 \%$ \\
\hline
\end{tabular}

Fuente: Cuestionario de la EES 2009 para España (Instituto Nacional de Estadística).

En el caso de las mujeres, las dos posiciones de no convivencia (ni en pareja ni con hijos) en las edades 30-49 registran valores inferiores a los de los hombres, mientras que en las edades 50-64 los valores femeninos son superiores. Esta diferencia se puede explicar por el incremento progresivo del número de mujeres que viven solas con la edad, debido al mayor nivel de supervivencia femenina combinado con su menor edad a la hora de casarse. Otra diferencia respecto al perfil masculino es el mantenimiento del peso relativo conjunto de las tres posiciones en el hogar que implican convivencia en pareja (73\% en $30-39,79 \%$ en $40-49$, $74 \%$ en $50-59$ y $74 \%$ en $60-64$ ), en contraposición al patrón masculino por edad apuntado anteriormente.

En lo que obviamente coinciden hombres y mujeres es en el aumento del valor relativo de la convivencia con hijos entre los adultos jóvenes (hasta los 50 años) ya que este fenómeno está estrechamente ligado a la edad, sobre todo en la población femenina y debido a su calendario reproductivo, mientras que en los dos últimos grupos de edad el porcentaje de núcleos sin hijos llega casi a doblarse (un 29\% en el 50-59 por un 49\% en el 60-64), coincidiendo con la emancipación de los hijos según su ciclo vital. En cuanto a la monoparentalidad, esta es la posición en el hogar donde se detectan diferencias relativas mayores entre hombres y mujeres, siendo el porcentaje de mujeres en esta posición mucho más alto. Por último, los valores relativos de las otras posiciones en el hogar son los más bajos entre las mujeres, y similares a los de los hombres.

\section{CVRS según sexo y edad}

La CVRS muestra claras diferencias por sexo y edad (Figura 2). Los hombres presentan unos valores superiores a los femeninos a todas las edades y estas diferencias son siempre estadísticamente significativas.

Los valores de CVRS son menores a medida que observamos grupos de edad más mayores, siendo estas diferencias únicamente significativas entre las mujeres. Estas presentan tres grupos de edad derivados de las diferencias significativas de CVRS a partir de los intervalos de confianza (IC): el primer grupo que lo forman las más jóvenes (30-34); un segundo grupo formado por las mujeres entre 35 y 54 años; y por último, los dos grupos de edades más maduras (55-59 y 60-64).

\section{CVRS según posición en el hogar y sexo}

Las posiciones relacionadas con la no convivencia en pareja ni hijos (vivir solo/a o posición de hijo/a) muestran valores inferiores de CVRS (Figura 3). Entre los hombres, estos valores son significativamente diferentes respecto a los de las posiciones en el hogar que implican convivencia en pareja, mientras que entre las mujeres los IC no nos permiten afirmar que la diferencia sea significativa.

Como se esperaba, vivir en pareja se asocia positivamente con la CVRS entre los hombres aunque se observan pequeñas diferencias según la convivencia con hijos y el número de estos. Los valores de las mujeres que conviven en pareja muestran también una situación ventajosa respecto a las que viven solas o en un núcleo monoparental, aunque no existe evidencia estadística de diferencia con las que ocupan la posición de hija. Los valores de CVRS se incrementan ligeramente en función del número de hijos que conviven en el hogar. Resulta más evidente que entre las mujeres la situación menos ventajosa es la de pertenecer a un núcleo monoparental. El bajo número de hombres en esta situación provoca IC muy amplios, lo que imposibilita sacar conclusiones a partir de esta Figura.

Por último, las otras posibles posiciones en el hogar, donde básicamente se incluyen los individuos fuera del núcleo principal del hogar, se muestran como una de las situaciones dentro del hogar con valores más altos de CVRS en ambos sexos. 
Figura 2.

Valores medios de CVRS según sexo y grupos quinquenales de edad. España 2009.
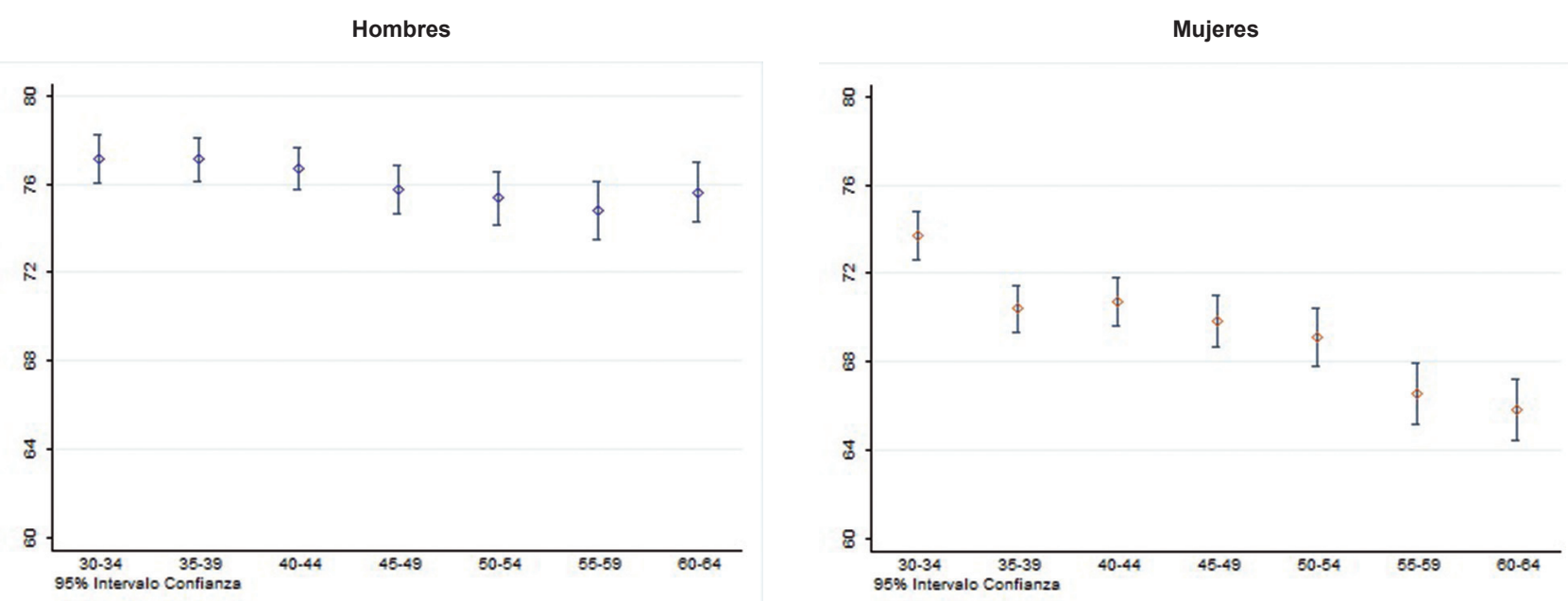

Fuente: Cuestionario de la EES 2009 para España (Instituto Nacional de Estadística).

\section{ANÁLISIS MULTIVARIABLE}

El análisis multivariable realizado para estimar la asociación neta entre la posición en el hogar y la CVRS se presenta de manera independiente para cada sexo en la tabla 3. Se controla por el efecto de los determinantes sociales mencionados anteriormente además de por la edad, la nacionalidad y los indicadores de salud objetiva.

Los coeficientes sin tipificar de la tabla 3 informan sobre el signo y la magnitud de la asociación entre las covariables introducidas y la variable dependiente, controlando por el efecto conjunto del resto de variables de cada modelo.
Los coeficientes tipificados permiten comparar el peso explicativo de cada variable dentro del modelo $y$, de este modo, saber qué variables tienen un mayor efecto sobre los cambios de las CVRS una vez eliminado el efecto de la diferente escala de medida de cada una de las variables independientes.

Para hombres y mujeres todas las posiciones dentro del hogar implican una CVRS más baja con respecto a la posición de referencia (vivir en pareja sin hijos) tal y como muestra el signo negativo de los respectivos coeficientes. En los hombres, vivir solo u ocupar la posición de hijo penalizan la CVRS en relación con los que viven en pareja sin hijos (coeficientes de $-3,64$ y $-4,46$ respectivamente).

Figura 3.

Media de CVRS por sexo y posición dentro del hogar. España 2009.

Hombres

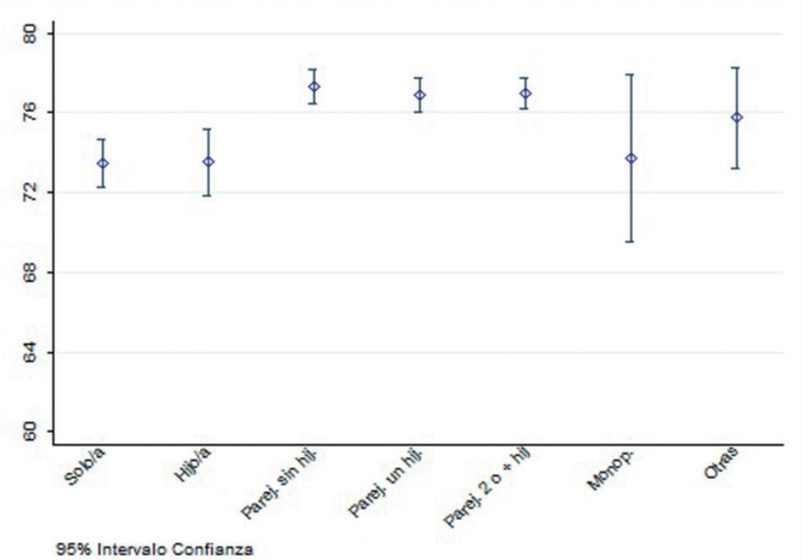

Mujeres

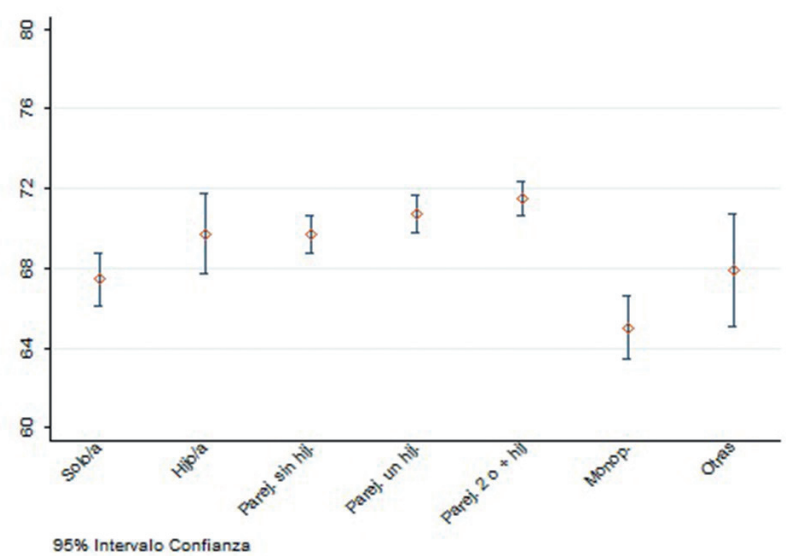

Fuente: Cuestionario de la EES 2009 para España (Instituto Nacional de Estadística). 
En este caso, al tratarse de las dos posiciones en el hogar relativas a la no convivencia en pareja o con hijos, cobra fuerza la idea de que la salud de los hombres es la que se ve más beneficiada por la convivencia en pareja (Lillard y Panis 1996; Waite 1995). Esto no es así entre las mujeres, ya que solo aquellas que viven solas muestran un coeficiente negativo y en este caso la significatividad estadística es baja (nivel 0,1 ).

Los resultados también introducen ciertos matices en relación con los patrones de CVRS esperados para hombres y mujeres. Por ejemplo, en relación con las posiciones en el hogar que implican tener hijos (pareja con un hijo, pareja con dos hijos o más y monoparentalidad) $y$, por tanto, un nivel de carga de cuidado superior. En estos casos se observa un efecto negativo y significativo en la CVRS entre los hombres. Por el contrario, entre las mujeres, únicamente aquellas que conviven con dos hijos 0 más 0 en situación de monoparentalidad se ven penalizadas con respecto a las que conviven en pareja sin hijos.

La pertenencia a un núcleo monoparental muestra una clara asociación negativa con la CVRS, siendo esta situación la que significativamente más rebaja sus valores tomando como referencia vivir en pareja sin hijos. La penalización es mayor entre las mujeres $(-6,23)$ que entre los hombres $(-4,20)$.

Los coeficientes tipificados revelan un papel importante de la posición en el hogar para entender la variabilidad de la CVRS dentro del conjunto de factores sociodemográficos analizados. Concretamente, la posición en el hogar es la tercera variable en cuanto a su capacidad para explicar la varianza del indicador de CVRS en el modelo femenino, por detrás de las variables de salud objetiva y de capital social y por delante de las variables socioeconómicas (relación con la actividad y nivel educativo) y demográficas (edad y nacionalidad). En el caso masculino, el peso explicativo de esta variable es similar al femenino, con la única diferencia de que la relación con la actividad presenta una capacidad explicativa superior.

Para los hombres, todas las posibles situaciones de la relación con la actividad significativas son de signo negativo, y por tanto, se asocian a valores de CVRS inferiores respecto a la categoría de referencia (asalariados). Estar desempleado, ser empresario con asalariados o formar parte de las otras tipologías de ocupación muestran valores menores de CVRS. Entre las mujeres se observa el mismo tipo de asociación entre las categorías significativas (inactivas, empresarias sin asalariados y otras personas ocupadas). Cabe señalar que el desempleo se muestra más perjudicial para la salud en los hombres que en las mujeres, entre las cuales esta situación no muestra diferencia significativa con las empleadas. Mención aparte merece el grupo de inactivos en ambos sexos, debido a la tipología de situaciones incluidas en esta categoría que de hecho guardan una estrecha relación con la salud (mayoritariamente personas jubiladas antes de los 65 años 0 incapacitadas). Esta resulta ser la situación de actividad que presenta mayor asociación negativa con la CVRS en ambos sexos incluso controlando mediante covariables de salud objetiva.

En relación con el nivel educativo, este tiene mayor poder explicativo en el modelo femenino que en el masculino. Las mujeres con estudios inferiores al nivel universitario presentan una asociación negativa y significativa con la CVRS en relación con las de nivel universitario. Entre los hombres esta asociación no resulta tan clara, ya que los individuos con estudios secundarios muestran mejores valores de CVRS respecto a la categoría de referencia (estudios universitarios).

El número de personas con las que se declara poder contar en caso de necesidad ante una hipotética situación grave muestra asociación de signo positivo tanto para hombres como para mujeres. Además, los coeficientes tipificados de esta variable son elevados. Es decir, que los individuos que declaran poder contar con más de dos personas presentan valores más altos de CVRS lo cual concuerda con resultados previos (Puga et al. 2007; Berkman et al. 2000).

La nacionalidad también se muestra como un factor diferenciador en relación con la variable dependiente y su efecto no es igual para hombres y mujeres. Entre los hombres, únicamente las nacionalidades extranjeras extracomunitarias presentan asociación significativa y de signo negativo respecto a aquellos que poseen nacionalidad española. En cambio, entre las mujeres esta asociación es significativa y de signo positivo entre las extranjeras en general. La heterogeneidad interna de este grupo poblacional según países de origen y tiempo de residencia en España invita a la cautela a la hora de formular hipótesis explicativas (García-Gómez y Oliva 2009).

La asociación entre CVRS y las dos variables de salud objetiva es la anticipada en trabajos anteriores donde se demuestra la estrecha relación entre las dimensiones objetiva y subjetiva de la salud (Damian et al. 1999). El dolor y las enfermedades crónicas muestran una asociación significativa de signo negativo con el valor de CVRS declarado, además de ser las variables con mayor capacidad explicativa en ambos modelos. El mayor beneficio analítico de incluir estas variables es controlar su efecto, obteniendo de este modo el valor de la asociación con la CVRS de los determinantes sociales y demográficos analizados a igual situación de salud objetiva. 
Tabla 3.

Coeficientes de los modelos de regresión lineal según sexo. España 2009.

\begin{tabular}{|c|c|c|c|c|c|c|c|}
\hline & & \multicolumn{3}{|c|}{ Hombres } & \multicolumn{3}{|c|}{ Mujeres } \\
\hline & & Coeficientes & $\begin{array}{l}\text { Coeficientes } \\
\text { tipificados }\end{array}$ & Sig. & Coeficientes & $\begin{array}{l}\text { Coeficientes } \\
\text { tipificados }\end{array}$ & Sig. \\
\hline & Constante & 81,98 & & $0,000 * * *$ & 81,91 & & 0,000 *** \\
\hline \multirow{6}{*}{$\begin{array}{l}\text { Posición dentro del Hogar } \\
\text { (Ref: Miembro pareja sin } \\
\text { hijos) }\end{array}$} & Vivir solo/a & $-3,64$ & $-0,06$ & $0,000 * * *$ & $-1,72$ & $-0,02$ & $0,068 \dagger$ \\
\hline & Posición Hijo/a en el núcleo & $-4,46$ & $-0,08$ & $0,000 * * *$ & $-0,92$ & $-0,01$ & 0,324 \\
\hline & Miembro pareja con un hijo/a & $-1,17$ & $-0,03$ & 0,042 * & $-0,87$ & $-0,02$ & 0,168 \\
\hline & Miembro pareja con dos o más hijos & $-1,96$ & $-0,05$ & $0,000 * * *$ & $-1,48$ & $-0,04$ & $0,015^{*}$ \\
\hline & Miembro núcleo monoparental & $-4,20$ & $-0,02$ & 0,040 * & $-6,23$ & $-0,08$ & $0,000 * * *$ \\
\hline & Otra Posibilidad & 0,94 & 0,01 & 0,388 & $-0,68$ & $-0,01$ & 0,557 \\
\hline \multirow{2}{*}{$\begin{array}{l}\text { Declara haber tenido dolor } \\
\text { o molestia física durante } \\
\text { las últimas } 4 \text { semanas } \\
\text { (Ref: No) }\end{array}$} & Dolor leve o moderado & $-5,81$ & $-0,15$ & $0,000 * * *$ & $-6,65$ & $-0,16$ & $0,000 * * *$ \\
\hline & Dolor severo o extremo & $-15,41$ & $-0,21$ & $0,000 * * *$ & $-15,68$ & $-0,27$ & $0,000 * * *$ \\
\hline \multirow{2}{*}{$\begin{array}{l}\text { Número de Enfermedades } \\
\text { Crónicas diagnosticadas } \\
\text { por un médico (Ref: } \\
\text { Ninguna) }\end{array}$} & Una & $-1,90$ & $-0,05$ & $0,000 * * *$ & $-2,93$ & $-0,07$ & $0,000 * * *$ \\
\hline & Dos o más & $-7,22$ & $-0,19$ & $0,000 * * *$ & $-10,21$ & $-0,26$ & $0,000 * * *$ \\
\hline \multirow{6}{*}{$\begin{array}{l}\text { Relación con la activi- } \\
\text { dad (Ref: Trabajador } \\
\text { asalariado/a) }\end{array}$} & Desempleado/a & $-3,33$ & $-0,07$ & 0,000 ** & $-0,29$ & $-0,01$ & 0,652 \\
\hline & Tareas domésticas & 6,23 & 0,02 & 0,132 & 0,39 & 0,01 & 0,504 \\
\hline & Inactivo/a & $-6,15$ & $-0,11$ & $0,000 * * *$ & $-5,98$ & $-0,08$ & $0,000 * * *$ \\
\hline & Empresario/a con asalariados & $-4,30$ & $-0,06$ & $0,000 * * *$ & $-1,33$ & $-0,01$ & 0,389 \\
\hline & Empresario/a sin asalariados & $-0,84$ & $-0,02$ & 0,219 & $-3,02$ & $-0,03$ & 0,003 ** \\
\hline & Otras personas ocupadas & $-4,25$ & $-0,03$ & $0,006 * *$ & $-2,90$ & $-0,02$ & $0,062 \dagger$ \\
\hline \multirow{2}{*}{$\begin{array}{l}\text { Nivel de estudios máximo } \\
\text { alcanzado (Ref: Estudios } \\
\text { universitarios) }\end{array}$} & Estudios obligatorios como máximo & 0,20 & 0,01 & 0,705 & $-3,40$ & $-0,09$ & $0,000 * * *$ \\
\hline & Estudios de secundaria no obligatorios & 1,39 & 0,04 & $0,012^{* *}$ & $-0,47$ & $-0,01$ & 0,419 \\
\hline $\begin{array}{l}\text { Número de personas con } \\
\text { las que cuenta en caso de } \\
\text { problema grave (Ref: Más } \\
\text { de dos) }\end{array}$ & Dos o menos & $-3,67$ & $-0,09$ & $0,000 * * *$ & $-5,78$ & $-0,13$ & $0,000 * * *$ \\
\hline \multirow{3}{*}{$\begin{array}{l}\text { Nacionalidad (Ref: } \\
\text { Nacionalidad española) }\end{array}$} & Nacionalidad extranjera (UE-27) & $-1,57$ & $-0,02$ & $0,085 \dagger$ & 2,58 & 0,03 & 0,017 * \\
\hline & Nacionalidad extranjera (Fuera UE-27) & $-2,36$ & $-0,04$ & $0,002 * *$ & 2,50 & 0,04 & 0,002 ** \\
\hline & Edad & 0,06 & 0,04 & 0,006 ** & 0,05 & 0,02 & $0,079 \dagger$ \\
\hline \multirow{2}{*}{\multicolumn{2}{|c|}{$\mathrm{R} 2$}} & & ,163 & & & 253 & \\
\hline & & & $* * *$ & $<0$, & $* *<0,01$ & $*<0,05$ & $t<0,1$ \\
\hline
\end{tabular}

Fuente: Cuestionario de la EES 2009 para España (Instituto Nacional de Estadística).

Finalmente, el coeficiente para la variable "edad" señala una asociación positiva y significativa en los hombres, mientras que en el caso femenino el coeficiente no resulta ser significativo aunque presenta el mismo signo. El signo positivo indica que cuando se controla mediante el resto de variables independientes, los hombres de mayor edad presentan valores de CVRS más elevados.

\section{CONCLUSIONES Y DISCUSIÓN}

Este trabajo analiza el papel de las principales posiciones en el hogar como determinante social de la salud a través del indicador de calidad de vida relacionada con la salud (CVRS) y controlando por el estatus socioeconómico, el capital social, la nacionalidad, la edad y la salud objetiva de los sujetos. Se analizan las edades 30-64 en las que las diferencias de género en la vida cotidiana y su impacto en la salud pueden ser más importantes como consecuencia de la etapa de ciclo familiar a la que comúnmente se asocia: la etapa de mayor carga productiva y reproductiva (Rosales 2003). La principal cualidad de la CVRS es su mayor sensibilidad para captar diferencias en salud en una población como la adulta (aquí edades 30-64) con relativamente poca heterogeneidad en salud objetiva. 
Este indicador contempla cuatro dimensiones de salud más psicológicas que fisiológicas (impacto emocional, cansancio psicológico, vitalidad y estado de ánimo). No obstante, los resultados muestran que esos aspectos no son independientes de las condiciones de salud objetiva, ajustándose de este modo a la multidimensionalidad del concepto de salud (Organización Mundial de la Salud 1946). Además, la CVRS presenta un patrón por sexo y edad muy similar al mostrado por otras medidas de la salud de naturaleza subjetiva, como la salud general autopercibida: mejores valores de salud para los hombres a cualquier edad, aunque las mujeres presenten mayores valores de supervivencia (Oksuzyan et al. 2009; Idler 1995). En relación con la edad, una vez controlado el efecto de los determinantes sociales de la salud, el aumento de la misma se relaciona con valores más elevados de CVRS. Esto, al igual que en el caso de la salud autopercibida (Gumà 2009), puede atribuirse a un proceso de normalización de la morbilidad en la vida cotidiana y/o al hecho del componente de selección positiva implícito en cualquier encuesta de salud (Carr et al. 2001). El efecto descrito de la edad sobre la CVRS es mayor entre la población masculina lo que podría explicarse en parte por un efecto de selección adicional de supervivencia (la población masculina está más seleccionada con la edad en términos de salud por su propia sobremortalidad). No obstante, estas diferencias entre hombres y mujeres en los niveles de CVRS van más allá de las simples diferencias biológicas entre sexos y se vinculan también a desigualdades de género (Observatorio de Salud de la Mujer 2005).

La posición en el hogar aproxima la estructuración de roles de género en relación tanto al ciclo de vida de los individuos como a los patrones de desigualdad en la distribución de las responsabilidades dentro de este ámbito. Los resultados muestran que esta variable tiene una capacidad altamente explicativa de las variaciones de la CVRS incluso estandarizando por el resto de determinantes sociales y demográficos analizados. Este hecho resulta consistente con lo hallado en trabajos previos que han mostrado la relevancia del hogar a la hora de explicar las desigualdades de salud (Martikainen et al. 2005). El peso explicativo de la posición en el hogar en relación con la CVRS es mayor en el caso femenino, donde su importancia se sitúa por encima de las variables de estatus socioeconómico individual. No ocurre lo mismo entre los hombres, poniendo de manifiesto la necesidad de superar la visión androcéntrica de la investigación en salud (Rohlfs, Borrell y Fonseca 2000; Griffith 2005; Ruíz y Verdú 2004). Tradicionalmente se ha centrado el análisis de las desigualdades en salud en el estatus socioeconómico (Artazcoz et al. 2004a; Rohlfs, Borrell y Fonseca 2000), obviando, por tanto, determinantes sociales de mayor relevancia a la hora de explicar las desigualdades de salud entre las mujeres.
Los resultados indican asimismo que la asociación entre la posición en el hogar y la CVRS se articula de manera diferente en hombres y mujeres, dependiendo de la relación familiar analizada (la convivencia en pareja y/o la paternidad-maternidad). La no convivencia en pareja afecta más negativamente la CVRS de los hombres en línea con trabajos anteriores (Lillard y Panis 1996; Waite 1995).

Un resultado interesante por lo inesperado del mismo es que cuando la convivencia en pareja se acompaña de hijo/s, se observa una reducción de los valores de CVRS más importante entre los hombres. Se ha destacado que convivir con hijos comporta una serie de beneficios para la salud por la reducción de actividades de riesgo vinculada a un mayor sentimiento de responsabilidad (Umberson 1987). No obstante, también lleva implícito un incremento de la carga de trabajo reproductivo que afecta al día a día de los individuos en aspectos como las horas de sueño, las pautas de alimentación, el estrés, etc. (Observatorio de Salud de la Mujer 2005). El hecho de que la CVRS de los hombres se vea afectada de manera más intensa por la convivencia con hijos, y ello estandarizando por la relación con la actividad y el nivel educativo, parece contradictorio con la tradicional mayor dedicación femenina al cuidado de estos. También resulta contradictorio con los efectos de la "doble presencia" derivada del rol de trabajadora y madre en relación con el incremento del estrés, la ansiedad y el malestar (Rosales 2003; Balbo 1978). Sin embargo, desde las teorías de construcción de las identidades de género puede explicarse este resultado como una consecuencia de la no asimilación o asimilación insuficiente de esa dualidad por parte de los hombres (Goldsheider 2000). El diferente valor dado en la construcción de la identidad masculina y femenina a la paternidad y la maternidad (Maclnnes 1998; García-Mina 2003), provoca una visión más positiva de las labores de crianza de los hijos por parte de las mujeres, elevando su tolerancia a este tipo de situaciones. Esto puede explicar que la CVRS entre las mujeres solo se vea afectada cuando se produce un incremento significativo de la carga reproductiva derivado de tener dos o más hijos.

Para ambos sexos la monoparentalidad es la posición del hogar asociada a una peor CVRS, siendo la que implica una mayor carga de trabajo reproductivo en comparación con todas las otras contempladas. Las consecuencias negativas de esta posición de hogar sobre la CVRS son mayores entre la población femenina, lo que podría estar relacionado con las dificultades derivadas de la ruptura del modelo patriarcal de familia con sustentador principal masculino, que deja en una situación de mayor vulnerabilidad económica a las mujeres (Madruga 2006; Bartfeld 2000). Este hecho puede condicionar la capacidad de poder delegar en el mercado algunas de las tareas de trabajo doméstico y de cuidado, incrementando de este modo el estrés procedente del hogar. Hay que tener en cuenta, además, que tras una situación de ruptura de pareja, la situación de monoparentalidad es mucho más frecuente entre las mujeres. 
La importancia de los roles de género a la hora de entender las desigualdades de salud en hombres y mujeres se ve confirmada en las diferencias encontradas en el resto de determinantes tenidos en cuenta, tanto en el sentido y la magnitud de la asociación, como en el peso relativo de cada uno de estos a la hora de explicar la variabilidad de CVRS de hombres y mujeres. Estas diferencias se observan de manera más acusada en relación con la actividad, el nivel educativo y la nacionalidad.

El estatus socioeconómico de los hombres se define básicamente a partir de la relación con la actividad según su papel de sustentador económico, mientras que entre las mujeres la educación juega también un papel importante junto a esta variable (Krieger et al. 1999). Por ejemplo, en el caso femenino resulta más relevante la influencia del nivel educativo sobre la CVRS a igualdad de situaciones de actividad. En cambio, los resultados indican que el desempleo penaliza significativamente la CVRS de los hombres y no la de las mujeres, lo que es coherente con los postulados teóricos de los roles de género y la construcción de su identidades. Estos otorgan mayor valor al empleo remunerado entre los hombres y, por tanto, su falta, perjudica especialmente la salud masculina.

Las mencionadas no son las únicas diferencias encontradas entre hombres y mujeres. Las categorías de empresario entre los hombres solo penalizan la CVRS entre los que tienen asalariados, lo que se puede presumir como una consecuencia del estrés que acompaña a esta situación laboral. Entre las mujeres, en cambio, las empresarias sin asalariados presentan una CVRS peor que las que tienen empleados a su cargo. En este último caso, dedicar un mayor número de horas al trabajo remunerado para gestionar una actividad empresarial propia no se da siempre de manera paralela a la reducción del tiempo destinado al trabajo doméstico (Artazcoz et al. 2004b). En cambio, cuando las empresarias cuentan con asalariados, presumiblemente estamos ante un colectivo que puede afrontar el coste económico de una delegación en el mercado remunerado de las tareas de cuidado que alivia la doble carga de trabajo total (ibíd.), lo cual podría explicar sus mejores valores de CVRS.

Existen, por último, indicios que alimentan el actual debate sobre si la asociación entre las relaciones en el hogar y las desigualdades de salud se deben a un efecto de selección (las probabilidades de encontrar pareja en el mercado matrimonial y/o de tener hijos dependen en parte del perfil de salud de los individuos) o de causalidad (la convivencia en pareja/hijos tiene beneficios directos sobre la salud). En general existe consenso en que ambos efectos influyen en esta asociación, pero sin que pueda concretarse el peso específico de cada uno de ellos en la misma (Joutsenniemi 2007; Lillard y Waite 1995). La naturaleza transversal del trabajo impide ahondar en el estudio de la selección. Sin embargo, el hecho de haber controlado mediante la salud objetiva y otros determinantes sociales de la salud permite indagar en el sentido de la causalidad. A igualdad de salud objetiva y perfil sociodemográfico asumimos estar ante individuos afectados por un nivel similar de selección previa por salud. Por tanto, las asociaciones descritas apuntan a una relación de causalidad entre la posición en el hogar y la dimensión subjetiva de la salud, tanto en el caso de la convivencia en pareja como en la carga del hogar derivada de convivir con hijos. Estos indicios deberán ser confirmados en futuros trabajos desde una óptica longitudinal.

\section{ReferenCias Bibliográficas}

Abraído-Lanza, A. F., B. P. Dohrenwend, D. S. Ng-Mak and J. B. Turner. 1999. "The Latino mortality paradox: A test of the "salmon bias" and healthy migrant hypotheses". American Journal of Public Health 89:1543-1548. http://dx.doi.org/10.2105/AJPH.89.10.1543

Arber, S. y H. Cooper. 1999. "Gender differences in health in later life: the new paradox?". Social Science and Medicine 48:61-76. http://dx.doi.org/10.1016/S0277-9536(98)00289-5

Anguita, J. C., J. R. Repullo y J. Pereira. 2001. "Medidas de calidad de vida relacionada con la salud. Conceptos básicos, construcción y adaptación cultural". Medicina Clínica 116:789-796. http://dx.doi.org/10.1016/S0025-7753(01)71987-2

Artazcoz, L., V. Escribà-Agüir y I. Cortès. 2004 a. "Género, trabajos y salud en España". Gaceta Sanitaria 18:24-35. http://dx.doi.org/10.1157/13061992

Artazcoz, L., L. Artieda, C. Borrell, I. Cortès, J. Benach y V. García. 2004 b. "Combining job and family demands and being healthy". European Journal of Public Health 14:43-48. http://dx.doi.org/10.1093/eurpub/14.1.43

Balbo, L. 1978. "La doppia presenza". Inchiesta 32:3-7.

Bartfeld, J. 2000. "Child support and the postdivorce economic well-being of mothers, fathers and children". Demography 37:203213. http://dx.doi.org/10.2307/2648122

Berkman, L. F., T. Glass, I. Brissette y T. E. Seeman. 2000. "From social integration to health: Durkheim in the new millennium". Social Sciences and Medicine 51:843-857. http://dx.doi.org/10.1016/S0277-9536(00)00065-4

Borrell, C., R. Peiró, N. Ramón, M. I. Pasarín, C. Colomer, E. Zafra y C. Álvarez-Dardet. 2004. "Desigualdades socioeconómicas y planes de salud en las comunidades autónomas del Estado espa-ol". Gaceta Sanitaria 19:277-286. http://dx.doi.org/10.1157/13078025

Bowling, A. 1999. "Health-related quality of life: A discussion of the concept, its use and measurement: the 'quality of life'". Biblioteca Virtual del Banco Mundial. Consulta 19 de noviembre 2012 (http://info.worldbank.org/etools/docs/library/48475/m2s5bowling.pdf).

Bullinger, M., R. Anderson, D. Cella y N. Aaronson. 1993. "Developing and evaluating cross-cultural instruments from minimum requirements to optimal models". Quality of Life Research 2:451-459. http://dx.doi.org/10.1007/BF00422219

Carr, A. J., B. Gibson y P. G. Robinson. 2001. "Measuring quality of life: Is quality of life determined by expectations or experience?". British Medical Journal 322:1240-1243. http://dx.doi.org/10.1136/bmj.322.7296.1240

Damian, J., A. Ruigómez, V. Pastor y J. M. Martín-Moreno. 1999. "Determinants of self assessed health among Spanish older 
people living at home". Journal Epidemiology and Community Health 53:412-416. http://dx.doi.org/10.1136/jech.53.7.412

Durán M. A. 1999. The future of work in Europe (Gendered patterns of time distribution). Bruselas: European Commission, Directorate General for Employment, Industrial Relations and Social Affairs.

García-Gómez, P. y J. Oliva. 2009. "Calidad de vida relacionada con la salud en población inmigrante en edad productiva". Gaceta Sanitaria 1:38-46. http://dx.doi.org/10.1016/j.gaceta.2009.09.008

García-Gómez, P. y J. Oliva. 2009. "Calidad de vida relacionada con la salud en población inmigrante en edad productiva". Gaceta Sanitaria 23:38-46. http://dx.doi.org/10.1016/j.gaceta.2009.09.008

García-Mina, A. 2003. Desarrollo del género en la feminidad y la masculinidad. Madrid: Narcea.

Goldsheider, F. K. 2000. "Men, children and the future of the family in the third millennium". The Futurist 32:527-538.

Griffith, D., K. Hamilton, J. Norrie y C. Isles. 2005. "Early and late mortality after myocardial infarction in men and women: prospective observational study". Heart 91:305-307. http://dx.doi.org/10.1136/hrt.2003.033035

Gumà, J. 2009. "Salud autopercibida en España: Análisis a partir de la Encuesta Nacional de Salud (1987-2006)". Master Thesis, Centre d'Estudis Demogràfics, Universitat Autònoma de Barcelona, Bellaterra.

Hughes, M. E. y L. J. Waite. 2002. "Health in household context: Living arrangements and health in late middle age". Journal of Health and Social Behavior 43:1-21. http://dx.doi.org/10.2307/3090242

Idler, E. L. 1993. "Age differences in self-assessments of health: age changes, cohort differences, or survivorship?". Journal of Gerontology 48:289-300. http://dx.doi.org/10.1093/geronj/48.6.S289

Idler, E. L. 1995. "Self-Ratings of Health: Do they also predict change in functional ability?". Journal of Gerontology 50:344-53. http://dx.doi.org/10.1093/geronb/50B.6.S344

Instituto Nacional de Estadística (INE). 2009. Encuesta Europea de Salud en España: Metodología. Madrid: INE. Consulta 10 de noviembre 2012 http://www.ine.es/metodologia/t15/t153042009.pdf

Joutsenniemi, K. 2007. "Living arrangements and health". PhD Dissertation. Department of Public Health. University of Helsinki, Helsinki.

Krieger, N., J. T. Chend y J. V. Selby. 1999. "Comparing individual-based and household-based measures of social class to assess class inequalities in women's health: a methodological study of 684 US women". Journal of Epidemiology and Community Health 53:612-623. http://dx.doi.org/10.1136/jech.53.10.612

Lillard, L., y L. Waite. 1995. "Til death do us part: Marital disruption and mortality". American Journal of Sociology 100:1131-1156. http://dx.doi.org/10.1086/230634

Lillard, L., y C. Panis. 1996. "Marital status and mortality: The role of health". Demography 33:313-327 http://dx.doi.org/10.2307/2061764

Madruga, I. 2006. Monoparentalidad y política familiar. Dilemas en torno a la madre cuidadora/madre trabajadora. Madrid: CIS.

Marmot, M. G. 2005. "Social determinants of health inequalities". The Lancet 365:1099-1104. http://dx.doi.org/10.1016/S01406736(05)71146-6

Maclnnes, J. 1998. The end of masculinity: The confusion of sexual genesis and sexual difference in modern society. Buckingham: Open University Press.

McDonald, P. 2000. "Gender equity in theories of fertility transition". Population and Development Review 26:427-440. http://dx.doi.org/10.1111/j.1728-4457.2000.00427.x
McKinlay, J. B. 1993. "The promotion of health through planned sociopolitical change: challenges for research and policy". Social Science and Medicine 36:109-117. http://dx.doi.org/10.1016/0277-9536(93)90202-F

Martikainen, P., T. Martelin, E. Nihtilä, K. Majamaa y S. Koskinen. 2005. "Differences in mortality by marital status in Finland from 1976 to 2000: Analyses of changes in marital-status distributions, socio-demographic and household composition, and cause of death". Population Studies 59:99-115. http://dx.doi.org/10.1080/0032472052000332737

Observatorio de Salud de la Mujer (OSM). 2005. Salud y Género 2005. Madrid: Ministerio de Sanidad y Consumo. Consulta 15 de mayo 2013. (http://www.msc.es/organizacion/sns/planCalidadSNS/pdf/equidad/InfomeSaludyGenero2005.pdf).

Oksuzyan, A., I. Petersen, H. Stovring, P. Bingley, J. W. Vaupel y K. Christensen. 2009. "The male-female health-survival paradox: A survey and register study of the impact of sex-specific selection and information bias". Annals of Epidemiology 19:504-511. http://dx.doi.org/10.1016/j.annepidem.2009.03.014

Organización Mundial de la Salud (OMS). 1946. Preámbulo de la Constitución de la Organización Mundial de la Salud en la Conferencia Internacional sobre Salud. New York, 19 junio-22 Julio.

Organización Mundial de la Salud (OMS). 2007. "A conceptual framework for action on the social determinants of health". Discussion paper for the Commission on Social Determinants of Health, April 2007. Consulta 15 de mayo 2013 http://www. who.int/social_determinants/resources/csdh_framework_action_05_07.pdf).

Porthé, V., M. Amable y J. Benach. 2006. "La precariedad laboral y la salud de los inmigrantes en España: ¿qué sabemos y qué deberíamos saber?" Archivos de prevención y riesgos laborales 10:34-39.

Puga, D., L. Rosero-Bixby, K. Glaser y T. Castro. 2007. "Red social y salud del adulto mayor en perspectiva comparada: Costa Rica, España e Inglaterra". Población y Salud en Mesoamérica 5:1-21. http://dx.doi.org/10.15517/psm.v5i1.4545

Ruiz, M. T. y M. Verdú. 2004. "Sesgo de género en el esfuerzo terapéutico". Gaceta Sanitaria 18:118-125. http://dx.doi.org/10.1157/13062260

Rogers, R. G. 1996. "The effects of family composition, health, and social support linkages on mortality". Journal on Health and Social Behavior 37:326-338. http://dx.doi.org/10.2307/2137260

Rohlfs, I., C. Borrell y M. C. Fonseca. 2000. "Género, desigualdades y salud pública: conocimientos y desconocimientos". Gaceta Sanitaria 14:60-71.

Rosales, R. M. 2003. Trabajo, salud y sexualidad: las cargas de trabajo laborales y reproductivas en la salud de las mujeres. Barcelona: Icaria Editorial.

Rose, G. 1992. Strategy of preventive medicine. Oxford: Oxford University Press.

Schneider, G., G. Driesch, A. Kruse, M. Wachter, H. Nehen y G. Heuft. 2004. "What influences self-perception of health in the elderly? The role of objective health condition, subjective wellbeing and sense of coherence". Archives of Gerontology and Geriatrics 39:227-237.

http://dx.doi.org/10.1016/j.archger.2004.03.005

Solé, M. y M. Rodríguez. 2010. "Disparidades entre inmigrantes y nativos en el impacto de las condiciones laborales en la salud". Gaceta Sanitaria 24:145-150. http://dx.doi.org/10.1016/j.gaceta.2009.10.006

Spijker, J. y J. Pérez. 2010. "La ocupación laboral de los convivientes con afectados por discapacidades: Un análisis multivariable". Revista Internacional de Sociología 68:311-332. http://dx.doi.org/10.3989/ris.2008.03.14 
Stephens, C. 1998. "The policy implications of health inequalities in developing countries". Pp. 288-307 en Human Biology and Social Inequality. 39th Symposium volume of the Society for the Study of Human Biology. Cambridge: Cambridge University Press.

Swallen, K. C. 1997. "Do health selection effects last? A comparison of morbidity rates for elderly adult immigrants and US-born elderly persons". Journal of Cross-Cultural Gerontology 12:317-339. http://dx.doi.org/10.1023/A:1006527615599

Umberson, D. 1987. "Family status and health behaviors: Social control effects as a dimension of social integration". Journal of Health and Social Behavior 28:306-319. http://dx.doi.org/10.2307/2136848

Vilagut, G., J. M. Valderas, M. Ferrer, O. Garin, E. López-García y J. Alonso. 2008. "Interpretación de los cuestionarios de salud SF-36 y SF-12 en Espa-a: componentes físico y mental". Medicina Clínica 130:726-735. http://dx.doi.org/10.1157/13121076

Waite, L. 1995. "Does marriage matter?". Demography 32:483-507. http://dx.doi.org/10.2307/2061670
ANTONIO D. CAMARA es Doctor en Historia Contemporánea por la Universidad de Granada. En la actualidad es investigador Ramón y Cajal en el departamento de Organización de Empresas, Marketing y Sociología de la Universidad de Jaén. Su investigación se centra en el gradiente socioeconómico del bienestar de la población a través de indicadores socio-sanitarios.

JORDI GUMÀ es becario FPI del Ministerio de Economía y Competitividad en el Centro de Estudios Demográficos. Anteriormente completó la European Doctoral School of Demography en Lund (Suecia). Su actual tema de investigación es la relación entre hogar y las desigualdades de salud subjetiva en España. El presente trabajo forma parte de su tesis doctoral inscrita en el doctorado de Demografía de la Universidad Autónoma de Barcelona.

ROCIO TREVIÑO es Doctora en Sociología por la Universitat Autònoma de Barcelona. Actualmente es investigadora en el Centro de Estudios Demográficos. Está especializada en el estudio de las nuevas formas familiares y de la dinámica demográfica en relación con la familia desde una perspectiva de género. 
AneXo

Tabla 1.

Descripción de la muestra de la EES 2009 por sexo. Recuento y valor medio de CVRS. España 2009 (no se han calculado los valores medios en categorías con menos de 20 casos).

\begin{tabular}{|c|c|c|c|c|c|c|}
\hline & \multicolumn{3}{|c|}{ Hombres } & \multicolumn{3}{|c|}{ Mujeres } \\
\hline & Recuento & Porcentaje & Media & Recuento & Porcentaje & Media \\
\hline Vivir solo/a & 511 & $7,7 \%$ & 73,36 & 413 & $6,3 \%$ & 68,87 \\
\hline Posición hijo/a en el núcleo & 661 & $9,9 \%$ & 73,78 & 475 & $7,2 \%$ & 71,76 \\
\hline Miembro pareja sin hijos & 1417 & $21,3 \%$ & 77,25 & 1369 & $20,7 \%$ & 70,36 \\
\hline Miembro pareja con un hijo/a & 1582 & $23,8 \%$ & 76,89 & 1555 & $23,5 \%$ & 70,91 \\
\hline Miembro pareja con dos o más hijos & 2070 & $31,1 \%$ & 76,46 & 1968 & $29,8 \%$ & 71,81 \\
\hline Miembro núcleo monoparental & 61 & $0,9 \%$ & 73,39 & 443 & $6,7 \%$ & 64,51 \\
\hline Otra posición hogar & 272 & $4,1 \%$ & 77,96 & 268 & $4,1 \%$ & 70,83 \\
\hline Identificación no posible & 81 & $1,2 \%$ & 78,98 & 112 & $1,7 \%$ & 67,35 \\
\hline Total & 6655 & $100 \%$ & 76,29 & 6603 & $100 \%$ & 70,5 \\
\hline No dolor últimas 4 semanas & 4585 & $68,9 \%$ & 79,33 & 3606 & $54,6 \%$ & 76,41 \\
\hline Dolor moderado últimas 4 semanas & 1683 & $25,3 \%$ & 71,72 & 2154 & $32,6 \%$ & 66,87 \\
\hline Dolor extremo últimas 4 semanas & 386 & $5,8 \%$ & 60,18 & 841 & $12,7 \%$ & 54,3 \\
\hline $\mathrm{Ns} / \mathrm{Nc}$ & 1 & $0,0 \%$ & - & 2 & $0,0 \%$ & - \\
\hline Total & 6655 & $100 \%$ & 76,29 & 6603 & $100 \%$ & 70,5 \\
\hline No enfermedad crónica & 3043 & $45,7 \%$ & 80,03 & 2439 & $36,9 \%$ & 77,86 \\
\hline Una enfermedad crónica & 1758 & $26,4 \%$ & 77,19 & 1552 & $23,5 \%$ & 73,64 \\
\hline Dos o más enfermedades crónicas & 1854 & $27,9 \%$ & 69,27 & 2612 & $39,6 \%$ & 61,72 \\
\hline $\mathrm{Ns} / \mathrm{Nc}$ & 0 & $0,0 \%$ & - & 0 & $0,0 \%$ & - \\
\hline Total & 6655 & $100 \%$ & 76,29 & 6603 & $100 \%$ & 70,5 \\
\hline Desempleado/a & 1020 & $15,3 \%$ & 73,53 & 955 & $14,5 \%$ & 69,79 \\
\hline Tareas domésticas & 14 & $0,2 \%$ & 80,34 & 1522 & $23,1 \%$ & 70,25 \\
\hline Inactivo/a & 683 & $10,3 \%$ & 68,75 & 449 & $6,8 \%$ & 58,43 \\
\hline Asalariado/a & 3836 & $57,6 \%$ & 78,28 & 3121 & $47,3 \%$ & 72,74 \\
\hline Empresario/z con asalariados & 366 & $5,5 \%$ & 74,44 & 120 & $1,8 \%$ & 73,47 \\
\hline Empresario/a sin asalariados & 620 & $9,3 \%$ & 77,94 & 308 & $4,7 \%$ & 67,79 \\
\hline Otras personas ocupadas & 109 & $1,6 \%$ & 73,24 & 124 & $1,9 \%$ & 69,21 \\
\hline $\mathrm{Ns} / \mathrm{Nc}$ & 7 & $0,1 \%$ & - & 4 & $0,1 \%$ & - \\
\hline Total & 6655 & $100 \%$ & 76,29 & 6603 & $100 \%$ & 70,5 \\
\hline Estudis obligatorios como máximo & 3197 & $48,0 \%$ & 75,19 & 3132 & $47,4 \%$ & 67,31 \\
\hline Estudios de secundaria & 2124 & $31,9 \%$ & 77,56 & 1991 & $30,2 \%$ & 72,51 \\
\hline Estudios universitarios & 1324 & $19,9 \%$ & 77 & 1479 & $22,4 \%$ & 74,52 \\
\hline $\mathrm{Ns} / \mathrm{Nc}$ & 10 & $0,2 \%$ & - & 1 & $0,0 \%$ & - \\
\hline Total & 6655 & $100 \%$ & 76,29 & 6603 & $100 \%$ & 70,5 \\
\hline $\begin{array}{l}\text { No cuenta con más de } 2 \text { personas con las cuáles } \\
\text { puede contar en caso de problema grave }\end{array}$ & 1433 & $21,5 \%$ & 72,65 & 1416 & $21,4 \%$ & 65,17 \\
\hline $\begin{array}{l}\text { Cuenta con más de } 2 \text { personas con las cuáles puede } \\
\text { contar en caso de problema grave }\end{array}$ & 5191 & $78,0 \%$ & 77,34 & 5163 & $78,2 \%$ & 71,98 \\
\hline $\mathrm{Ns} / \mathrm{Nc}$ & 31 & $0,5 \%$ & 67,86 & 24 & $0,4 \%$ & 68,22 \\
\hline Total & 6655 & $100 \%$ & 76,29 & 6603 & $100 \%$ & 70,5 \\
\hline Nacionalidad española & 5790 & $87,0 \%$ & 76,42 & 5816 & $88,1 \%$ & 70,11 \\
\hline Nacionalidad extranjera (EU-27) & 335 & $5,0 \%$ & 75,64 & 266 & $4,0 \%$ & 74,44 \\
\hline Nacionalidad extranjera (Fuera EU-27) & 528 & $7,9 \%$ & 75,29 & 519 & $7,9 \%$ & 72,88 \\
\hline $\mathrm{Ns} / \mathrm{Nc}$ & 2 & $0,0 \%$ & - & 2 & $0,0 \%$ & - \\
\hline Total & 6655 & $100 \%$ & 76,29 & 6603 & $100 \%$ & 70,5 \\
\hline
\end{tabular}

* Esta categoría muestra valores significativamente inferiores al resto dentro de la misma variable. Sin embargo, la dificultad para interpretar el significado de dicha categoría ha hecho que no sea incluida en el modelo multivariable

Fuente: Elaboración propia, a partir de los microdatos de la muestra española de la EES 2009. 
Tabla 2.

Coeficientes de los modelos de regresión lineal global. España 2009.

\begin{tabular}{|c|c|c|c|c|}
\hline & & Coeficientes & $\begin{array}{l}\text { Coeficientes } \\
\text { tipificados }\end{array}$ & Sig. \\
\hline & Constante & 83,91 & & $0,000 * * *$ \\
\hline Sexo (Ref: Hombres) & Mujeres & $-3,30$ & $-0,09$ & 0,000 *** \\
\hline \multirow{6}{*}{ Posición dentro del Hogar (Ref: Miembro pareja sin hijos) } & Vivir solo/a & $-2,90$ & $-0,04$ & $0,000 * * *$ \\
\hline & Posición Hijo/a en el núcleo & $-2,86$ & $-0,04$ & 0,000 *** \\
\hline & Miembro pareja con un hijo/a & $-0,95$ & $-0,02$ & $0,027^{*}$ \\
\hline & Miembro pareja con dos o más hijos & $-1,56$ & $-0,04$ & $0,000^{* * *}$ \\
\hline & Miembro núcleo monoparental & $-6,31$ & $-0,07$ & $0,000 * * *$ \\
\hline & Otra Posibilidad & 0,13 & 0,00 & 0,868 \\
\hline \multirow{2}{*}{$\begin{array}{l}\text { Declara haber tenido dolor o molestia física durante las últimas } \\
4 \text { semanas (Ref: No) }\end{array}$} & Dolor leve o moderado & $-6,31$ & $-0,16$ & $0,000 * * *$ \\
\hline & Dolor severo o extremo & $-15,80$ & $-0,25$ & $0,000 * * *$ \\
\hline \multirow{2}{*}{$\begin{array}{l}\text { Número de Enfermedades Crónicas diagnosticadas por un } \\
\text { médico (Ref: Ninguna) }\end{array}$} & Una & $-2,29$ & $-0,05$ & $0,000 * * *$ \\
\hline & Dos o más & $-8,86$ & $-0,23$ & $0,000 * * *$ \\
\hline \multirow{6}{*}{ Relación con la actividad (Ref: Trabajador asalariado/a) } & Desempleado/a & $-1,94$ & $-0,04$ & $0,000^{* * *}$ \\
\hline & Tareas domésticas & $-0,60$ & $-0,01$ & 0,248 \\
\hline & Inactivo/a & $-5,63$ & $-0,09$ & $0,000 * * *$ \\
\hline & Empresario/a con asalariados & $-3,32$ & $-0,03$ & $0,000 * * *$ \\
\hline & Empresario/a sin asalariados & $-1,45$ & $-0,02$ & $0,012^{*}$ \\
\hline & Otras personas ocupadas & $-3,74$ & $-0,03$ & 0,001 ** \\
\hline \multirow{2}{*}{$\begin{array}{l}\text { Nivel de estudios máximo alcanzado (Ref: Estudios universita- } \\
\text { rios) }\end{array}$} & Estudios obligatorios como máximo & $-1,68$ & $-0,05$ & $0,000 * * *$ \\
\hline & Estudios de secundaria no obligatorios & 0,37 & 0,01 & 0,354 \\
\hline $\begin{array}{l}\text { Número de personas con las que cuenta en caso de problema } \\
\text { grave (Ref: Más de dos) }\end{array}$ & Dos o menos & $-4,69$ & $-0,11$ & $0,000 * * *$ \\
\hline \multirow{3}{*}{ Nacionalidad (Ref: Nacionalidad española) } & Nacionalidad extranjera (UE-27) & 0,27 & 0,00 & 0,705 \\
\hline & Nacionalidad extranjera (Fuera UE-27) & $-0,12$ & 0,00 & 0,835 \\
\hline & Edad & 0,05 & 0,03 & 0,004 ** \\
\hline R2 & & & ,225 & \\
\hline & $* * \star$ & ${ }^{* *}<0,01$ & ${ }^{*}<0,05$ & $\dagger<0,1$ \\
\hline
\end{tabular}

Fuente: Elaboración propia, a partir de los microdatos de la muestra española de la EES 2009. 